\title{
A Rare Case of Metastatic Heterogeneous Poorly Differentiated Neuroendocrine Carcinoma of lleum: A Case Report and Literature Review
}

\author{
Andrei Nikiforchin ${ }^{\mathrm{a}}$, Ruth Peng ${ }^{\mathrm{b}}$, Michelle Sittig ${ }^{\mathrm{a}}$, Sandy Kotiah ${ }^{\mathrm{c}, \mathrm{d}}$
}

\begin{abstract}
Neuroendocrine neoplasms (NENs) represent a diverse group of tumors arising from neuroendocrine cells. Current World Health Organization (WHO) classification is based on tumor differentiation and grade defined by mitotic rate and/or Ki-67 index to determine prognosis and treatment options. However, some NENs do not meet WHO pathology criteria due to morphologic heterogeneity and this leads to management challenges. WHO defines poorly differentiated NENs of the gastrointestinal tract as having morphologically large or small cell features with marked elevation of Ki-67. We present a unique clinical case that does not fit either growth pattern and also has heterogeneity with a well-differentiated component. This case report and literature review highlights the current limitations of the WHO classification of small bowel NENs and the subsequent challenges in management decisions for the patients.
\end{abstract}

Keywords: Neuroendocrine neoplasm; Neuroendocrine carcinoma; Tumor grade; Morphologic heterogeneity; WHO classification

\section{Introduction}

The small bowel is the most common location of neuroendocrine neoplasms (NENs) $[1,2]$. The majority of small bowel NENs are found in the ileum and typically represent welldifferentiated low-grade (G1) histopathologic type, whereas poorly differentiated high-grade (G3) type is a quite rare finding [1, 3-7]. Despite the favorable prognosis in G1 histology,

Manuscript submitted December 14, 2019, accepted December 20, 2019

aDepartment of Surgical Oncology, Mercy Medical Center, 227 Saint Paul Place, Baltimore, MD, USA

bepartment of Pathology, Mercy Medical Center, 345 Saint Paul Place, Baltimore, MD, USA

'Department of Medical Oncology and Hematology, Mercy Medical Center, 227 Saint Paul Place, Baltimore, MD, USA

${ }^{\mathrm{d} C}$ Corresponding Author: Sandy Kotiah, Department of Medical Oncology and Hematology, Mercy Medical Center, 227 Saint Paul Place, Baltimore, MD, USA.Email: skotiah@mdmercy.com

doi: https://doi.org/10.14740/jmc3401 approximately $48 \%$ of patients with ileum NENs present with distant disease at diagnosis and $14-17 \%$ have peritoneal carcinomatosis (PC) [1, 2, 8-10].

Currently, the treatment of advanced ileum NENs varies from surgical options, liver-directed therapy, everolimus and somatostatin analogues for G1-G2 well-differentiated neuroendocrine tumors (NETs), to systemic chemotherapy for G3 poorly differentiated neuroendocrine carcinomas (NECs) [11, 12]. Therefore, the grade and differentiation of a tumor in accordance with the current World Health Organization (WHO) classification is an important step for clinical decision making in these patients $[5,7]$. However, several studies have shown that gastroenteropancreatic (GEP) NENs can demonstrate heterogeneity in their proliferative rate/grade between the primary site and metastases $[13,14]$. This heterogeneity leads to challenges in diagnosis and appropriate treatment options.

In this report, we present a rare case of metastatic heterogeneous ileum NEC, accompanied by a literature review.

\section{Case Report}

A 67-year-old African American woman with an unremarkable medical, social and family history underwent an incisional hernia repair. During surgery, PC was incidentally found with

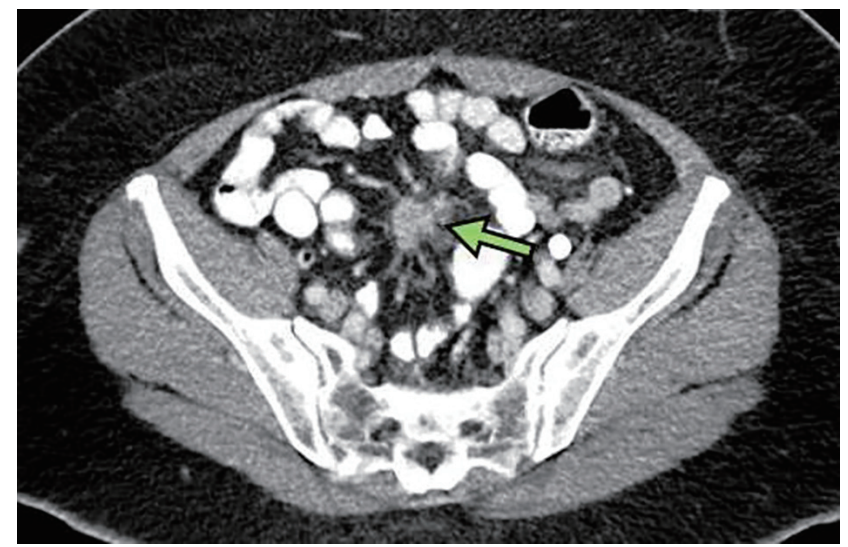

Figure 1. Abdominal CT scan of mass (arrow) involving mesentery compatible with a neuroendocrine tumor measuring approximately 2.1 $\times 1.8 \mathrm{~cm}$ in orthogonal axial dimensions and up to $3.1 \mathrm{~cm}$ in craniocaudal extent. CT: computed tomography. 

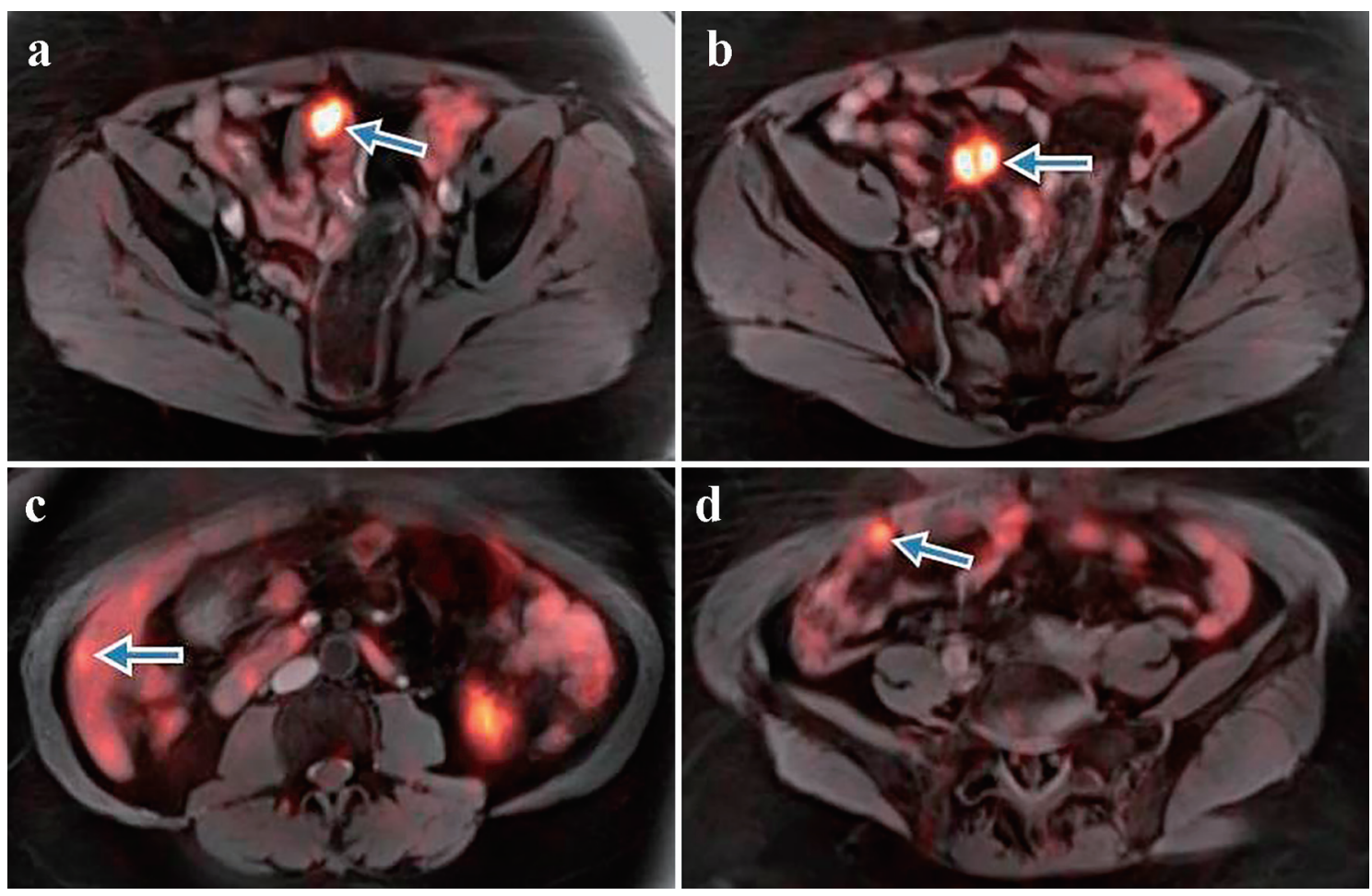

Figure 2. (a) Ga-68 DOTATATE PET-MRI shows a prominent somatostatin avid lesion (arrow) measuring $1.9 \times 2.8 \mathrm{~cm}$ with SUV 28 within a loop of distal small bowel. (b) Well-defined $2.2 \times 2.1 \mathrm{~cm}$ lesion (arrow) with prominent tracer uptake (SUV 22.3) adjacent to the small bowel mesentery. (c) Ga-68 PET-MRI displays a tiny focus (arrow) in segment 6 of the liver consistent with liver metastasis. (d) Small peritoneal lesion (arrow) with a high tracer uptake consistent with peritoneal carcinomatosis. Ga-68 DOTATATE PET-MRI: positron emission tomography-magnetic resonance imaging with gallium-68; SUV: standardized uptake value.

final biopsy histopathology revealing a well-differentiated G1 NET with Ki-67 $<3 \%$ and mitotic rate of $<1$ per 10 high-power fields (HPF). Preoperative computed tomography (CT) revealed a mesenteric mass measuring $2.1 \times 1.8 \times 3.1 \mathrm{~cm}$ (Fig. 1 ) and a single right hepatic lobe lesion measuring $1.1 \times 1.0 \mathrm{~cm}$. Gallium-68 (Ga-68) DOTATATE positron emission tomography-magnetic resonance imaging (PET-MRI) described a 1.9 $\times 2.8 \mathrm{~cm}$ lesion in the small bowel, a $2.2 \times 2.1 \mathrm{~cm}$ mesenteric nodule, one small liver lesion and several peritoneal lesions with active tracer uptake (Fig. 2a-d). The patient complained of frequent bowel movements daily during previous 6 months. A 24-h urine 5-hydroxyindoleacetic acid (5-HIAA) level was elevated to $10.6 \mathrm{mg}$. Other laboratory results were within normal limits.

Somatostatin analog therapy was initiated due to diarrhea and elevated 5-HIAA. The case was discussed in multidisciplinary tumor board and the patient underwent cytoreductive surgery (CRS). The primary tumor site was the terminal ileum with five small liver metastases as well as extensive peritoneal involvement. The peritoneal cancer index was 26 and a complete cytoreduction was achieved (CC-0).

Postoperative pathology reported a heterogeneous poorly differentiated G3 NEC (Fig. 3a, b) with perineural invasion (Fig. 4), regional lymph node metastases, liver and peritoneal involvement. The Ki-67 index varied within the tumor, ranging from $3 \%$ to $25 \%$ (Fig. $5 \mathrm{a}, \mathrm{b}$ ) and up to 19 mitotic figures per 10 HPF (Fig. 6). Features were not consistent with either small or large cell type histology. Molecular testing did not yield any targeted mutations or immunotherapy markers. The patient is currently undergoing treatment with somatostatin analog for her functional tumor and is asymptomatic without any signs of recurrence at 6-month follow-up.

\section{Discussion}

This patient was initially diagnosed after peritoneal biopsy with a resectable metastatic G1 ileum NET. One of the current standards of care for metastatic well-differentiated G1-G2 NETs is surgical resection when feasible to achieve remission $[11,12,15]$. Surgical approach is offered to patients with liver metastases when debulking of $\geq 70 \%$ of the liver metastases is possible [16-18]. Retrospective data also state that CRS provides a survival benefit in selected patients with PC of welldifferentiated NETs origin, while the addition of hyperthermic intraperitoneal chemotherapy (HIPEC) does not improve outcomes $[10,19]$. The patient underwent extensive CRS without HIPEC and the final surgical histopathology revealed a poorly differentiated G3 NEC with several non-typical features.

There have been many attempts to establish a classification of NENs covering all tumor subtypes in an effort to clarify treatment options and prognosis. However, the creation and clinical application of NENs classification remains challenging due to significant heterogeneity of these tumors $[14,20]$. The 

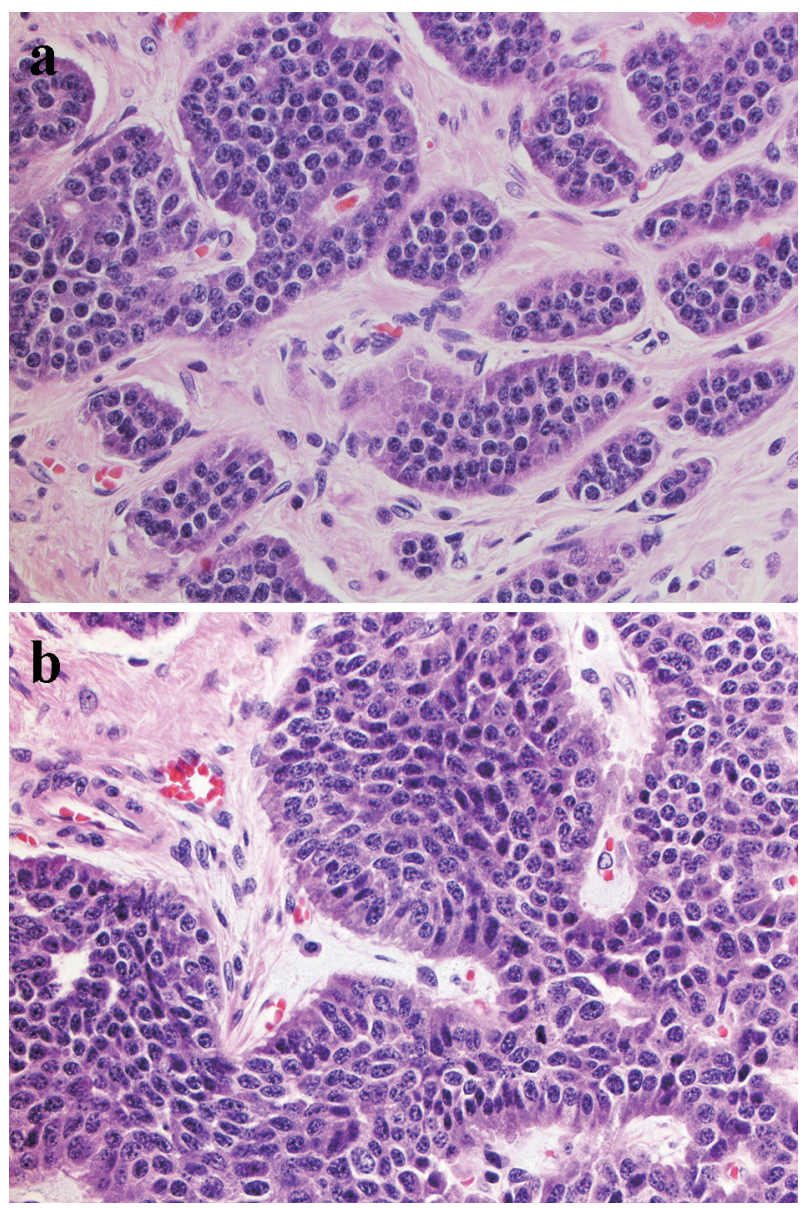

Figure 3. Morphologic presentation of poorly differentiated G3 NEC Tumor cells are polygonal with moderate amount of cytoplasm with larger size than in small cell carcinoma, but smaller than in large cell NEC. The nuclei are pleomorphic, the chromatin is finely granular and nucleoli are seen. (a) Tumor area consistent with low Ki-67 (3$5 \%)$ (H\&E, × 100 magnification); (b) Tumor area with high Ki-67 (25\%) (H\&E, $\times 100$ magnification). NEC: neuroendocrine carcinoma; H\&E: hematoxylin \& eosin.

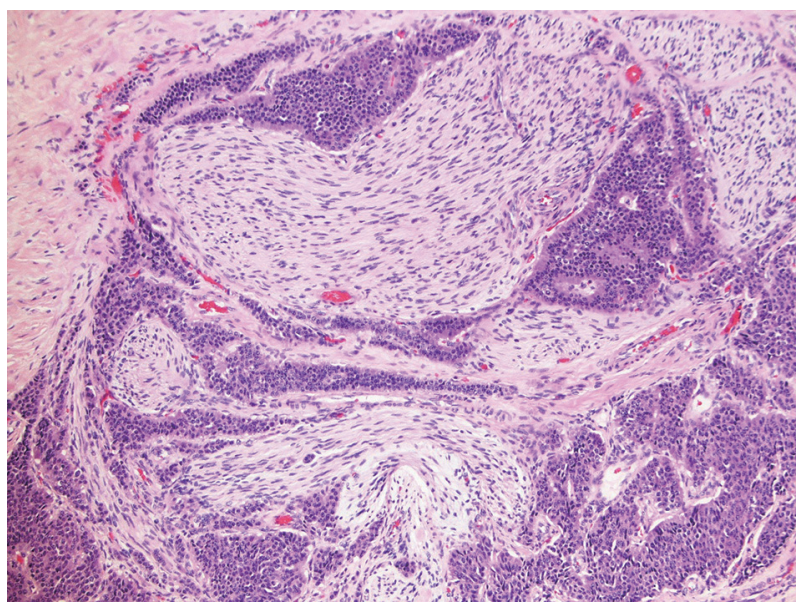

Figure 4. Perineural invasion in primary ileal NEC $(\mathrm{H} \& \mathrm{E}, \times 40$ magnification). NEC: neuroendocrine carcinoma; H\&E: hematoxylin \& eosin.
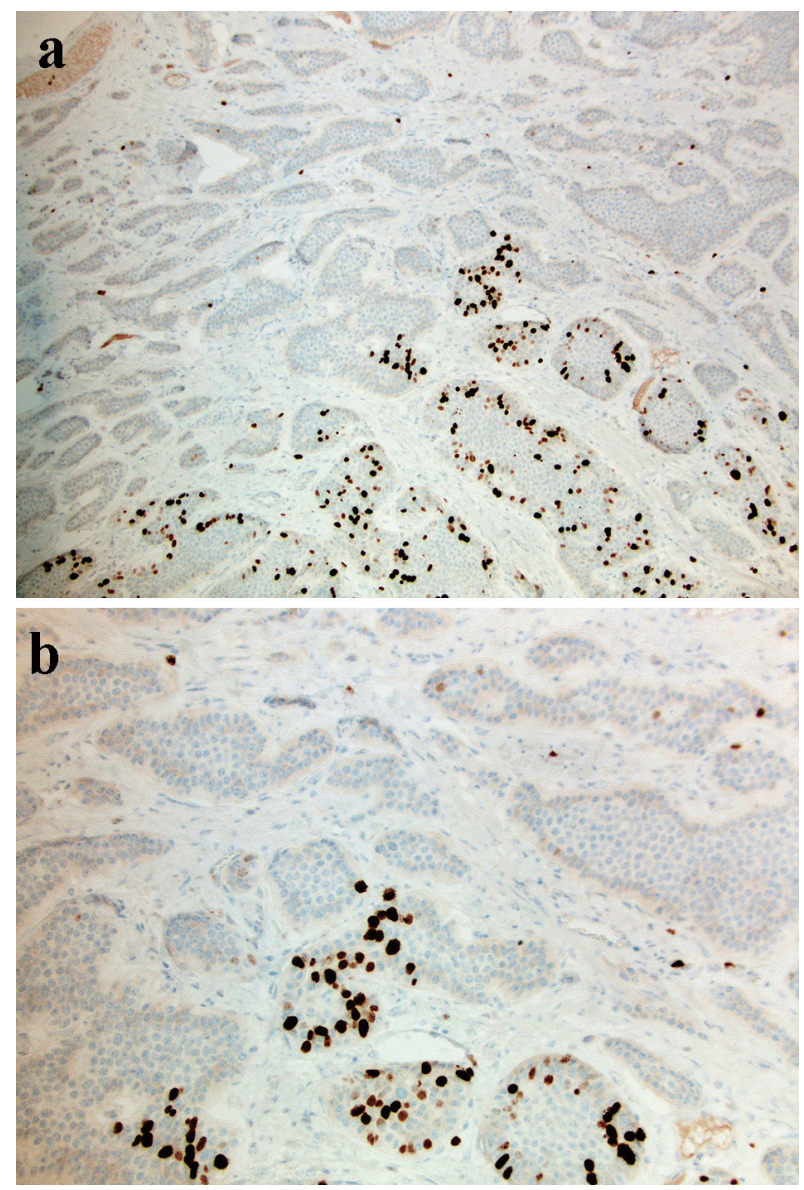

Figure 5. A Ki-67 stain shows primary tumor with various expression of Ki-67 ranging 3-25\% (a: $\times 40$ magnification; b: $\times 100$ magnification).

most recent updated version of WHO classification is based on cell differentiation, mitotic rate and $\mathrm{Ki}-67$ index [7, 21]. It divides all GEP NENs into four groups: G1 NET, G2 NET, small cell G3 NEC and large cell G3 NEC [5, 7]. The subdivision

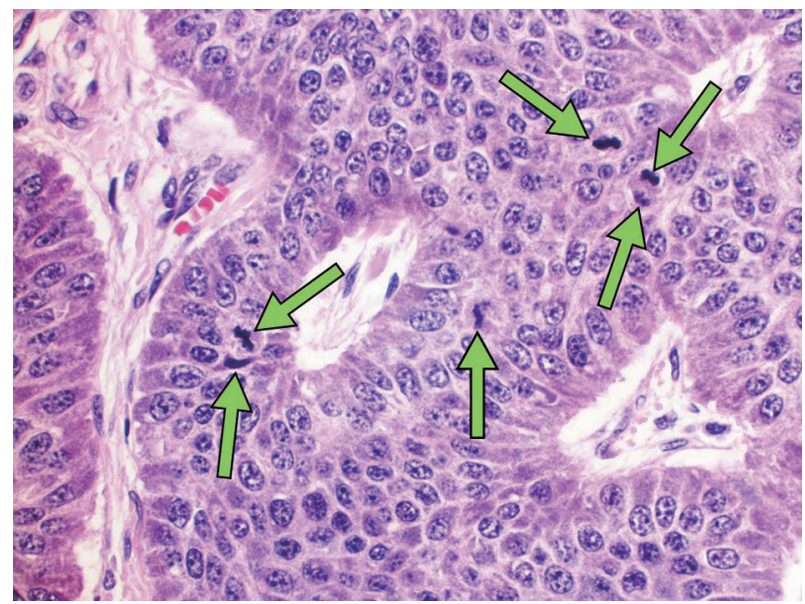

Figure 6. Mitotic tumor activity. Arrows identify mitotic figures in the nuclei of primary tumor cells (H\&E, $\times 200$ magnification). H\&E: hematoxylin \& eosin. 
of small and large cell G3 NECs arose from lung NECs due to similar morphology, tumor behavior and treatment response [22-24]. Although the tumor in this case had G3 NEC features, neither small nor large cell histology was identified. This is an example of how the current classification does not encompass all tumor types, making final determination and treatment direction ambiguous when these rare instances are encountered.

Tumor heterogeneity is an obstacle for proper tumor classification in a particular NENs subgroup; however, this is not a rare phenomenon in well-differentiated NETs [25]. Several previous reports showed that mitotic rate and $\mathrm{Ki}-67$ index can vary within one specimen and between the primary and metastases in GEP NENs $[14,26]$. Tang et al reported in their retrospective review of 31 GEP G1 NET cases that the G3 component occurred in $48 \%$ of the primary tumors and in $52 \%$ of metastatic sites [13]. In contrast, tumor heterogeneity is quite uncommon in poorly differentiated NECs which typically present with consistent $\mathrm{Ki}-67>20 \%$ [27]. In the present case, postoperative assessment of the primary tumor revealed a G3 NEC with Ki-67 up to $25 \%$ (Fig. 5a, b). However, a preoperative biopsy from the metastatic lesion showed a well-differentiated G1 NET with Ki-67 $<3 \%$ and mitotic rate of $<1$ per 10 HPF. Therefore, it might be reasonable to consider a biopsy of different metastatic sites, but this is not always feasible [13, 25]. Without surgical exploration, it can be difficult to identify the primary tumor, therefore serial imaging can help determine if the tumor biology is concordant with the pathology results.

The clinical decisions in heterogeneous tumors are difficult as they cannot be based only on tumor grade. Expression of somatostatin receptors (SRs) status, which defines the efficacy of treatment with somatostatin analogs and peptide receptor radionuclide therapy, might be helpful in the management of this group of patients. Historically, high expression of SRs is a common diagnostic indicator of well-differentiated G1-G2 NETs, whereas poorly differentiated G3 NECs express few, if any, SRs [28, 29]. Therefore, somatostatin analogs are usually preferred for G1-G2 NETs and uncommonly administered in patients with G3 NEC [12]. However, several studies reported that up to $40-50 \%$ G3 NECs demonstrate active tracer uptake at SR-based imaging (particularly Ga-68 DOTATATE PET) $[20,30,31]$. Potentially, functional imaging can be used for prediction of response to somatostatin analog therapy based on the activity of tracer uptake [27]. In Koch et al's study, the administration of octreotide demonstrated longer progression-free survival in NET patients who had higher tracer uptake (standardized uptake value $(\mathrm{SUV})>20.3)$ on Ga-68 DOTATATE PET compared to patients with low SUV (69 and 26 weeks, respectively) [32]. In the present case, Ga-68 DOTATATE PET-MRI also visualized several lesions with SRs (Fig. 2a-d) with maximal SUV of 28. This allowed us to consider long-acting somatostatin analogs for treatment, despite G3 NEC pathology.

In contrast, chemotherapy has shown effectiveness in the treatment of poorly differentiated G3 NECs and no benefit in patients with G1-G2 NETs, making treatment decisions of heterogeneous tumors challenging $[11,12]$. In addition, several studies indicate that the efficacy of chemotherapy varies within G3 tumors. Velayoudom-Cephise et al showed no treatment response to cisplatin-based chemotherapy in patients with well- differentiated G3 NETs in comparison with a 31\% rate of response in patients with G3 NECs [20]. Another study showed worse response to chemotherapy in $15 \%$ of patients with $\mathrm{Ki}-67$ $<55 \%$, while treatment response with $\mathrm{Ki}-67>55 \%$ was seen in $42 \%$ [33]. The data demonstrate that the G3 group might be heterogeneous in clinical behavior and management of these tumors should not be based solely on tumor grade. In the present case, final pathology showed a poorly differentiated tumor with maximal Ki-67 of $25 \%$ and multiple foci of Ki-67 $<20 \%$. The current clinical guidelines have not provided treatment algorithms for poorly differentiated G3 NECs tumors with a low proliferative rate $[11,12]$. However, considering existing data, we assumed that the pathology characteristics of this tumor were not favorable for significant treatment response. Additional data are clearly needed to help guide the management of G3 patients with a low proliferative rate.

Molecular testing of GEP NENs can be a promising tool for choosing treatment options. For instance, pancreatic NEC patients with mutated KRAS and loss of Rb showed a much higher response rate for platinum-based chemotherapy than those with wild-type KRAS and retained Rb (100\% vs. $18 \%$, respectively) [34]. Despite a lack of data about the correlation between specific mutations and treatment response in small bowel NENs, there are some genetic mutations that can be helpful for clinical management. In a systematic review of 33 retrospective studies and eight case reports, the molecular features of GEP NECs were assessed and the presence of TP53 mutation was reported in $57-100 \%$ and loss of $\mathrm{Rb}$ was detected in $44-56 \%$ of cases [35]. Although there were no molecular alterations in the present case, we believe molecular testing might be useful for NETs to determine additional treatment options.

Current WHO classification is designed to guide treatment and prognosis for all NENs. Unfortunately, because of heterogeneity of these neoplasms, physicians are challenged to treat patients with tumors that cannot be defined to any of the known NEN subtypes at this current time. Considering all clinical and diagnostic data, we decided that the patient was a good candidate for long-acting octreotide for diarrhea symptom management. We hope that it will also help with tumor control, but that will be hard to determine as she has no measurable disease at this time. The patient is being closely monitored and further treatment options will be determined based on pattern and timing of recurrence.

\section{Conclusion}

This case report demonstrates the diagnostic and treatment challenges of rare heterogeneous small bowel poorly differentiated NEC. We believe that our experience can help to better clarify the challenges of the current WHO classification and clinical trial design for these patients in the future.

\section{Acknowledgments}

None to declare. 


\section{Financial Disclosure}

Authors have no grants and financial support to disclose.

\section{Conflict of Interest}

Andrei Nikiforchin, Ruth Peng and Michelle Sittig declare that they have no competing interests. Sandy Kotiah reports being a speaker for Novartis and Ipsen.

\section{Informed Consent}

The signed informed consent was obtained from the patient before conducting the study.

\section{Author Contributions}

Sandy Kotiah and Andrei Nikiforchin had the idea for the concept of this manuscript. Andrei Nikiforchin designed the paper, made literature search and wrote the first manuscript draft. Ruth Peng reviewed the pathology specimens and provided the images. Sandy Kotiah and Michelle Sittig reviewed the manuscript, and edited it for English, stylistics and grammar. Sandy Kotiah performed the final manuscript review. All authors contributed to the review and amendments of the manuscript for important intellectual content and approved the final version for submission.

\section{Data Availability}

The authors declare that data supporting the findings of this study are available within the article.

\section{References}

1. Niederle MB, Hackl M, Kaserer K, Niederle B. Gastroenteropancreatic neuroendocrine tumours: the current incidence and staging based on the WHO and European Neuroendocrine Tumour Society classification: an analysis based on prospectively collected parameters. Endocr Relat Cancer. 2010;17(4):909-918.

2. Amin MB, Greene FL, Edge SB, Compton CC, Gershenwald JE, Brookland RK, Meyer L, et al. The Eighth Edition AJCC Cancer Staging Manual: Continuing to build a bridge from a population-based to a more "personalized" approach to cancer staging. CA Cancer J Clin. 2017;67(2):93-99.

3. Maggard MA, O'Connell JB, Ko CY. Updated population-based review of carcinoid tumors. Ann Surg. 2004;240(1):117-122.

4. Norlen O, Stalberg P, Oberg K, Eriksson J, Hedberg J, Hessman O, Janson ET, et al. Long-term results of surgery for small intestinal neuroendocrine tumors at a tertiary re- ferral center. World J Surg. 2012;36(6):1419-1431.

5. Rindi G, Petrone G, Inzani F. The 2010 WHO classification of digestive neuroendocrine neoplasms: a critical appraisal four years after its introduction. Endocr Pathol. 2014;25(2):186-192.

6. Klimstra DS, Modlin IR, Coppola D, Lloyd RV, Suster S. The pathologic classification of neuroendocrine tumors: a review of nomenclature, grading, and staging systems. Pancreas. 2010;39(6):707-712.

7. Bosman F, Carneiro F, Hruban R. WHO classification of tumors of the digestive system. 4th edn Geneva. Switzerland: World Health Organization. 2010. https://scholar.google.ch/ scholar?oi=bibs\&hl=en\&cluster $=860650996615241074$.

8. Frilling A, Modlin IM, Kidd M, Russell C, Breitenstein S, Salem R, Kwekkeboom D, et al. Recommendations for management of patients with neuroendocrine liver metastases. Lancet Oncol. 2014;15(1):e8-21.

9. Lawrence B, Gustafsson BI, Chan A, Svejda B, Kidd M, Modlin IM. The epidemiology of gastroenteropancreatic neuroendocrine tumors. Endocrinol Metab Clin North Am. 2011;40(1):1-18, vii.

10. de Mestier L, Lardiere-Deguelte S, Brixi H, O'Toole D, Ruszniewski P, Cadiot G, Kianmanesh R. Updating the surgical management of peritoneal carcinomatosis in patients with neuroendocrine tumors. Neuroendocrinology. 2015;101(2):105-111.

11. Kunz PL, Reidy-Lagunes D, Anthony LB, Bertino EM, Brendtro K, Chan JA, Chen H, et al. Consensus guidelines for the management and treatment of neuroendocrine tumors. Pancreas. 2013;42(4):557-577.

12. Partelli S, Bartsch DK, Capdevila J, Chen J, Knigge U, Niederle B, Nieveen van Dijkum EJM, et al. ENETS consensus guidelines for standard of care in neuroendocrine tumours: surgery for small intestinal and pancreatic neuroendocrine tumours. Neuroendocrinology. 2017;105(3):255-265.

13. Tang LH, Untch BR, Reidy DL, O'Reilly E, Dhall D, Jih L, Basturk O, et al. Well-differentiated neuroendocrine tumors with a morphologically apparent highgrade component: a pathway distinct from poorly differentiated neuroendocrine carcinomas. Clin Cancer Res. 2016;22(4):1011-1017.

14. Basturk O, Yang Z, Tang LH, Hruban RH, Adsay V, McCall CM, Krasinskas AM, et al. The high-grade (WHO G3) pancreatic neuroendocrine tumor category is morphologically and biologically heterogenous and includes both well differentiated and poorly differentiated neoplasms. Am J Surg Pathol. 2015;39(5):683-690.

15. Howe JR, Cardona K, Fraker DL, Kebebew E, Untch BR, Wang YZ, Law CH, et al. The surgical management of small bowel neuroendocrine tumors: consensus guidelines of the North American neuroendocrine tumor society. Pancreas. 2017;46(6):715-731.

16. Lesurtel M, Nagorney DM, Mazzaferro V, Jensen RT, Poston GJ. When should a liver resection be performed in patients with liver metastases from neuroendocrine tumours? A systematic review with practice recommendations. HPB (Oxford). 2015;17(1):17-22.

17. Maxwell JE, Sherman SK, O'Dorisio TM, Bellizzi 
AM, Howe JR. Liver-directed surgery of neuroendocrine metastases: What is the optimal strategy? Surgery. 2016;159(1):320-333.

18. Scott AT, Breheny PJ, Keck KJ, Bellizzi AM, Dillon JS, O'Dorisio TM, Howe JR. Effective cytoreduction can be achieved in patients with numerous neuroendocrine tumor liver metastases (NETLMs). Surgery. 2019;165(1):166175.

19. Elias D, David A, Sourrouille I, Honore C, Goere D, Dumont F, Stoclin A, et al. Neuroendocrine carcinomas: optimal surgery of peritoneal metastases (and associated intra-abdominal metastases). Surgery. 2014;155(1):5-12.

20. Velayoudom-Cephise FL, Duvillard P, Foucan L, Hadoux J, Chougnet CN, Leboulleux S, Malka D, et al. Are G3 ENETS neuroendocrine neoplasms heterogeneous? Endocr Relat Cancer. 2013;20(5):649-657.

21. Inzani F, Petrone G, Rindi G. The New World Health Organization classification for pancreatic neuroendocrine neoplasia. Endocrinol Metab Clin North Am. 2018;47(3):463-470.

22. Basturk O, Tang L, Hruban RH, Adsay V, Yang Z, Krasinskas AM, Vakiani E, et al. Poorly differentiated neuroendocrine carcinomas of the pancreas: a clinicopathologic analysis of 44 cases. Am J Surg Pathol. 2014;38(4):437447.

23. La Rosa S, Sessa F. High-grade poorly differentiated neuroendocrine carcinomas of the gastroenteropancreatic system: from morphology to proliferation and back. Endocr Pathol. 2014;25(2):193-198.

24. Dasari A, Mehta K, Byers LA, Sorbye H, Yao JC. Comparative study of lung and extrapulmonary poorly differentiated neuroendocrine carcinomas: A SEER database analysis of 162,983 cases. Cancer. 2018;124(4):807-815.

25. Yang Z, Tang LH, Klimstra DS. Effect of tumor heterogeneity on the assessment of $\mathrm{Ki} 67$ labeling index in welldifferentiated neuroendocrine tumors metastatic to the liver: implications for prognostic stratification. Am J Surg Pathol. 2011;35(6):853-860.

26. Couvelard A, Deschamps L, Ravaud P, Baron G, Sauvanet $\mathrm{A}$, Hentic $\mathrm{O}$, Colnot $\mathrm{N}$, et al. Heterogeneity of tumor prognostic markers: a reproducibility study applied to liver metastases of pancreatic endocrine tumors. Mod Pathol. 2009;22(2):273-281.

27. Fazio N, Milione M. Heterogeneity of grade 3 gastroenteropancreatic neuroendocrine carcinomas: New in- sights and treatment implications. Cancer Treat Rev. 2016;50:61-67.

28. Barrio M, Czernin J, Fanti S, Ambrosini V, Binse I, Du L, Eiber M, et al. The Impact of Somatostatin ReceptorDirected PET/CT on the Management of Patients with Neuroendocrine Tumor: A Systematic Review and MetaAnalysis. J Nucl Med. 2017;58(5):756-761.

29. Oberg K, Kvols L, Caplin M, Delle Fave G, de Herder W, Rindi G, Ruszniewski P, et al. Consensus report on the use of somatostatin analogs for the management of neuroendocrine tumors of the gastroenteropancreatic system. Ann Oncol. 2004;15(6):966-973.

30. Heetfeld M, Chougnet CN, Olsen IH, Rinke A, Borbath I, Crespo G, Barriuso J, et al. Characteristics and treatment of patients with G3 gastroenteropancreatic neuroendocrine neoplasms. Endocr Relat Cancer. 2015;22(4):657664.

31. Raj N, Valentino E, Capanu M, Tang LH, Basturk O, Untch BR, Allen PJ, et al. Treatment response and outcomes of Grade 3 pancreatic neuroendocrine neoplasms based on morphology: well differentiated versus poorly differentiated. Pancreas. 2017;46(3):296-301.

32. Koch W, Auernhammer CJ, Geisler J, Spitzweg C, Cyran $\mathrm{CC}$, Ilhan H, Bartenstein P, et al. Treatment with octreotide in patients with well-differentiated neuroendocrine tumors of the ileum: prognostic stratification with Ga68-DOTA-TATE positron emission tomography. Mol Imaging. 2014;13:1-10

33. Sorbye H, Welin S, Langer SW, Vestermark LW, Holt $\mathrm{N}$, Osterlund P, Dueland S, et al. Predictive and prognostic factors for treatment and survival in 305 patients with advanced gastrointestinal neuroendocrine carcinoma (WHO G3): the NORDIC NEC study. Ann Oncol. 2013;24(1):152-160.

34. Hijioka S, Hosoda W, Matsuo K, Ueno M, Furukawa M, Yoshitomi H, Kobayashi N, et al. Rb loss and KRAS mutation are predictors of the response to platinum-based chemotherapy in pancreatic neuroendocrine neoplasm with Grade 3: a Japanese multicenter pancreatic NEN-G3 study. Clin Cancer Res. 2017;23(16):4625-4632.

35. Girardi DM, Silva ACB, Rego JFM, Coudry RA, Riechelmann RP. Unraveling molecular pathways of poorly differentiated neuroendocrine carcinomas of the gastroenteropancreatic system: A systematic review. Cancer Treat Rev. 2017;56:28-35. 